\title{
Konstruktion eines Inserts \\ für Faserverbund- Halbzeuge
}

Frank Weidermann, Stefanie Zimmermann, Andrea Pino

Sandwichplatten sind effiziente Leichtbauelemente. Sie werden im Fahrzeugbau, in der Luft- und Raumfahrt, im Werkzeugmaschinenbau und überall dort, wo es auf geringe bewegte Massen ankommt, eingesetzt. Sandwichplatten sind oft Halbzeuge. Sandwichbauteile haben oft einen aufwendigen Herstellungsprozess, der eine Wärmebehandlung, die höhere Genauigkeiten ausschließt, beinhaltet. Aus diesen Gründen ist es oft sinnvoll, Inserts erst im Nachhinein in die fertige Sandwichplatte bzw. in das fertige Sandwichbauteil einzusetzen. Diese Inserts haben unterschiedliche Aufgaben. Sie dienen z.B. der Aufnahme von Schrauben, Bolzen und Lagern. Die vorgestellte Verbindung entspricht durch ihre spezielle Geometrie den hohen Anforderungen für Verbindungselemente in den genannten Gebieten hinsichtlich Genauigkeit und Stabilität. Es wird der Konstruktionsprozess eines Inserts für Sandwichplatten aus CFK-Verbundmaterial beschrieben. Ausgehend von einer patentierten Lösung der Autoren findet eine schrittweise Produktverbesserung bis hin zu einem einsatzfähigen Prototypen statt. Durch die Insertverbindung können für den Maschinenbau erforderliche Genauigkeiten nun mit CFK- Halbzeugen umgesetzt werden. Die Anwendung dieses Leichtbaupotentials führt zur Energieeinsparung und zum Transfer von CFK- Anwendungen in den Maschinenbau.

Keywords: Insert, Faserverbund- Halbzeuge, Leichtbau, Sandwichplatten, Genauigkeit

\section{Problembeschreibung}

Sandwichplatten haben viele Vorteile beim Leichtbau. Ihr prinzipieller Aufbau ist vergleichbar mit einem Doppel T-Träger, wobei der Abstandshalter bei der Sandwichplatte die Funktion des Steges beim Doppel T-Träger einnimmt. Als Abstandshalter für eine CFK-Sandwichplatte kommen leichte Materialien wie Wabenstrukturen aus Papier und Aluminium oder auch geschäumte Materialien in Betracht. Der Herstellungsprozess von CFK-Sandwichplatten ist sehr komplex und aufwendig. So ist es für die allermeisten Anwender sinnvoll Halbzeuge zu kaufen und weiterzuverarbeiten, genauso wie die meisten Anwender Blech kaufen und sich kein eigenes Walzwerk zulegen. In der Regel erfolgt die Aushärtung der CFK-Platten durch eine Wärmebehandlung, z. B. im Autoklaven. Bei diesem Prozess geht Genauigkeit verloren, vergleichbar mit der Verformung durch den Wärmeeintrag beim Schweißen. Um CFK-Sandwichplatten dennoch 
für Strukturbauteile im Maschinenbau einzusetzen, ergeben sich folgende Anforderungen:

- Die Insertverbindung muss in die fertige CFK-Sandwichplatte eingebracht werden um Genauigkeit, z.B. durch CNC-Bearbeitung zu erreichen und um Halbzeuge verwenden zu können.

- Die Insertverbindung muss Kräfte in die Sandwichplatte einleiten können, die zur Sandwichplatte und zum anschließenden Maschinenelement passen. Eine Kette ist immer nur so stark, wie ihr schwächstes Glied.

\section{Stand der Technik und Vorbetrachtungen}

\section{Inserts für Faserverbund- Sandwichstrukturen}

Im Patent GB 2017857 wird ein Insert für Sandwichplatten vorgestellt, welches aus zwei Flanschen besteht, die gegeneinander verschraubt werden. Durch die Schraubverbindung wird von beiden Seiten auf die Sandwichplatte Druck ausgeübt, welcher das Kernmaterial der Sandwichplatte zusammendrückt. Distanzkörper zum Abbau dieses Druckes und zum Schutz des Kernmaterials sind nicht vorgesehen. Im Patent EP 1937985B1 wird ein Insert für Sandwichplatten mit Wabenkern vorgestellt, bei dem Bauteile, ähnlich einer Spinne, in den Wabenkern hineinragen und dort eine Verbindung herstellen. Es ist keine Deck- und Bodenplatte vorgesehen, die die Sandwichplatte zusammendrückt. Dieses Insert ist für wenig belastete Bauteile wie zum Beispiel die Gepäckklappen in Flugzeugkabinen geeignet. In den Patenten DE 3841499 A1, GB 2214594 A und EP 2294324 (A1) werden Schraubverbindungen vorgestellt, die dadurch gekennzeichnet sind, dass durch das Anziehen der Schraube an die Seitenflächen des Loches gepresst werden. Diese Verbindungen sind für Sandwichplatten nicht geeignet, weil sie ein stabiles Kernmaterial benötigen.

In einer Veröffentlichung von Heimbs, \& Pein wird eine Zusammenfassung aller bekannten Lösungen von Inserts für Sandwichplatten gegeben. Dabei sind neben den beiden schon beschriebenen Lösungen, die auch noch nachträglich eingebaut werden können, folgende Lösungen zu nennen.

Das Insert wird von einer Seite an die Sandwichplatte anlaminiert. Diese Lösung ist eigentlich für Bauteile ohne Sandwichstruktur besser geeignet, siehe Abbildung $1 \mathrm{~g}$.

In der Sandwichplatte werden schon bei der Herstellung an die Stellen wo Inserts angebracht werden sollen anstatt des Kernmaterials Blockmaterial (Holz, Aluminium, Stahl, Plastik) eingesetzt. An diesen Stellen kann dann direkt ein bzw. durchgeschraubt 
werden bzw. ein Insert gemäß Patent GB 2017857 verwendet werden. Mit dieser Lösung können stabile Verbindungen erreicht werden. Anstatt des Blockmaterials ist es auch möglich die Sandwichplatten an einer Seite aufzubohren, das Kernmaterial zu entfernen und Vergussmasse einzufüllen.

Die letzte vorgestellte Lösung ist an Stellen, wo Inserts eingebaut werden sollen punktuell von der Sandwichstruktur abzuweichen. Diese muss auch schon bei der Herstellung des Sandwichbauteils eingeplant werden.

Es soll davon ausgegangen werden, als Halbzeug hergestellte Sandwichplatte zu verwenden. Bei diesen sind alle Lösungen, die das Einlegen von Blockmaterial bzw. anderweitige konstruktive Veränderungen an der Sandwichplatte erfordern nicht möglich.

a

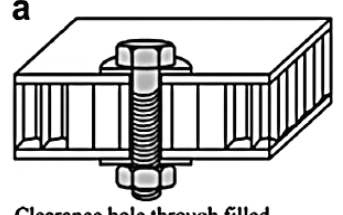

Clearance hole through filled honeycomb cells (potting mass)

e

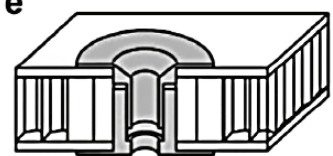

Double flanged bushing in filled honeycomb cells

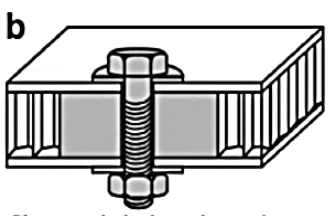

Clearance hole through massive block (e.g. metal, plastic, wood)

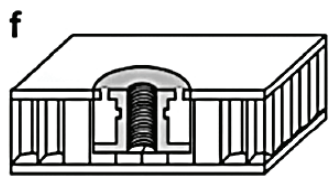

Threaded insert, glued into filled honeycomb cells

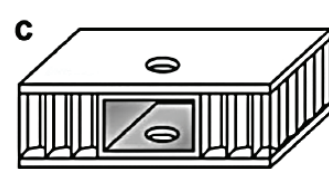

Clearance hole through metallic hollow profile

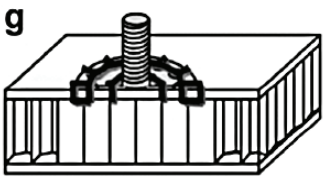

Threaded onsert, stitched onto the upper composite skin

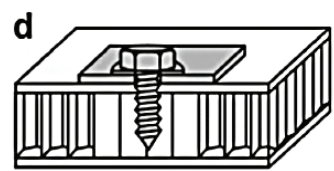

Blind hole in filled honeycomb cells (potting mass)

h

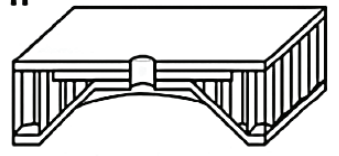

Monolithic load introduction area by merging upper and lower skin

Abbildung 1: Inserts für Wabensandwichplatten [1]

Die im Patent GB 2017857 vorgestellte Lösung, die der Erfindung am ähnlichsten ist, hat den Nachteil, dass durch die Schraubverbindung Druck auf das Kernmaterial ausgeübt wird. Bei leichtem und weichem Kernmaterial, was ja der Sinn einer Sandwichplatte ist, kann so keine feste Verschraubung des Inserts mit der Sandwichplatte erreicht werden. Einseitig an die Sandwichplatte auflaminierte bzw. eingeleimte Inserts erreichen auch keine Stabilität.

\section{Fertigungsgerechte Konstruktion}

Beim Gestalten von Bauteilen aus CFK ist es genau wie bei anderen Fertigungsverfahren und Materialien wichtig auf eine fertigungs- und materialgerechte Konstruktion zu achten. In der nachfolgenden Tabelle soll das am Beispiel der Verbindung von zwei 
Rohren verdeutlicht werden. Für Rohrverbindungen aus CFK-Rohren wäre die Klebverbindung die am besten geeignete Art der Verbindung. Es ist wichtig nicht einfach die Konstruktionen zu übernehmen, die für andere Fertigungsverfahren und Materialien geeignet sind, sondern die materialspezifischen Eigenschaften vorteilbringend auszunutzen.

Konstruktionsbestimmende Eigenschaften von CFK sind:

- Sehr hohe Zugfestigkeit ( $\sigma z=2000$ MPa für UD- Material sind möglich),

— Im Vergleich zur Zugfestigkeit hohe Wechselfestigkeit (über 80 \% der Zugfestigkeit),

- Dichte bei etwa 1,6 g/cm3

- Der Wärmeausdehnungskoeffizient ist durch gezielten Lagenaufbau einstellbar,

- Das Material ist mechanisch bearbeitbar und lässt sich gut kleben,

- Der Faserverlauf soll nicht durch scharfe Kanten/ Umlenkungen gestört werden,

- Ein aufwendiger Herstellungsprozess, so dass häufig die Verwendung von Halbzeugen sinnvoll ist.

Aus diesen Eigenschaften ergeben sich für CFK besondere Vorteile beim Einsatz für Leichtbauanwendungen.

\section{Vorgehen beim Entwickeln und Konstruieren nach VDI 2221}

In der inzwischen zurück gezogenen VDI-Richtlinie 2221 vom Mai 1993 „Methodik zum Entwickeln und Konstruieren technischer Systeme und Produkte" wird der Konstruktions- und Entwicklungsprozess mit sieben Arbeitsschritten beschrieben. Nach jedem Arbeitsschritt liegt ein Arbeitsergebnis vor. Der Konstruktions- und Entwicklungsprozess wird für verschiedene Anwendungen wie z. B. Verfahrensentwicklung oder Software-Entwicklung beschrieben. Jeder Arbeitsschritt wird erläutert. Beispiele dienen zur Veranschaulichung. So hat sich dieser Konstruktions- und Entwicklungsprozess viele Jahre bewährt, ihr Inhalt findet sich in vielen Lehrbüchern der Konstruktionslehre wieder.

Im Jahre 2019 wurde die VDI-Richtlinie 2221 durch eine Nachfolgeversion ersetzt. Der Titel ist jetzt: „Entwicklung technischer Produkte und Systeme - Modell der Produktentwicklung" (Blatt 1) und "Entwicklung technischer Produkte und Systeme - Gestaltung individueller Produktentwicklungsprozesse" (Blatt 2). 
In der neuen Richtlinie findet man die Arbeitsschritte bei der Produktentwicklung in der Spalte Aktivitäten des Gesamtschaubildes wieder. Es gibt auch Ergebnisse. Diese sind aber nicht zwingend den Arbeitsschritten zugeordnet.

Man findet diese Dinge nur wieder, wenn man die Vorgängerrichtlinie kennt. (Meinung des Autors)

Zwischen der Spalte Aktivitäten und der Spalte Ergebnisse ist noch eine Spalte mit Phasen. Diese sieht aus wie ein kleinteiliger Zeitplan. Zusammenfassend ist festzustellen, dass beide Richtlinien einen ähnlichen Inhalt haben, die Version vom Mai 1993 ist klarer und eindeutiger und bietet sich zur Anwendung an.

Tabelle 1: Fertigungsgerechte Gestaltung am Beispiel einer Rohrverbindung

$\begin{array}{ll}\begin{array}{l}\text { Fertigungs- } \\ \text { verfahren }\end{array} & \begin{array}{l}\text { Erklärung } \\ \text { Sie Fase sorgt dafür, dass der } \\ \text { Klebstoff nicht weggeschoben } \\ \text { wird. Beim Fügen Bauteile dre- } \\ \text { hen. Längere Überlappung zur } \\ \text { Übertragung der Kräfte. }\end{array} \\ \text { Kleben } & \begin{array}{l}\text { Längere Überlappung zur Über- } \\ \text { tragung der Kräfte. Dünne Spalte } \\ \text { damit das Lot durch die Kapillar- } \\ \text { wirkung gut fließt. }\end{array} \\ \text { Schweißen } & \begin{array}{l}\text { V-Naht wegen der Zugänglichkeit } \\ \text { beim Schweißen. Stumpfstoß } \\ \text { ausreichend, weil Rohr und } \\ \text { Schweißnaht annähernd die glei- } \\ \text { che Festigkeit haben. }\end{array}\end{array}$




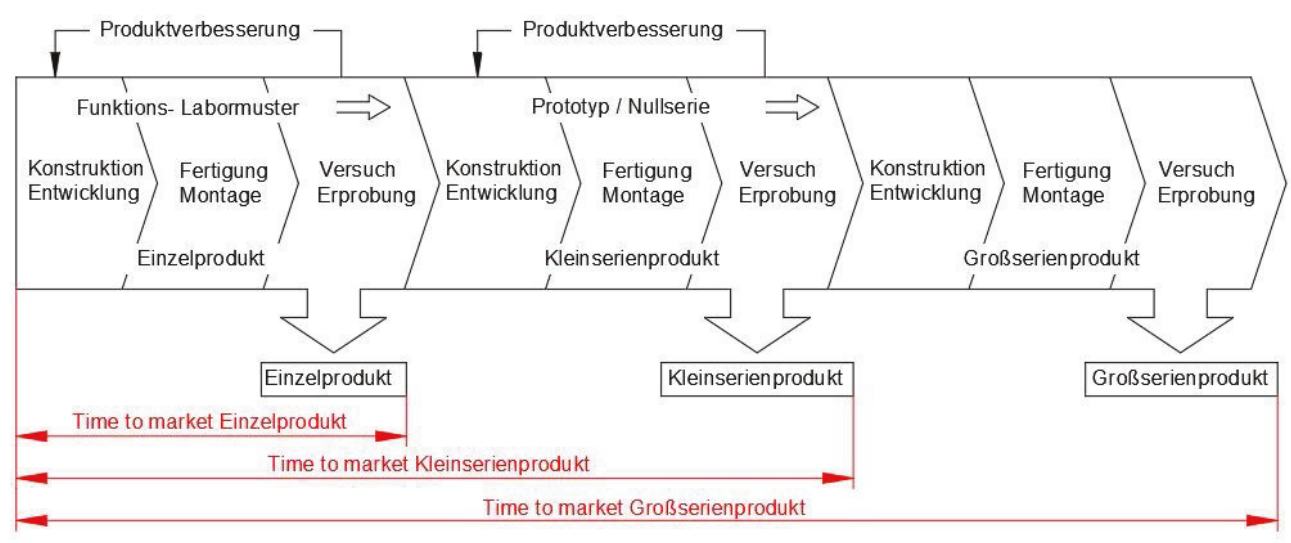

Abbildung 2: Phasen der Produktentstehung nach VDI 2221

\section{Lösungsfindung für die Insertverbindung nach VDI 2221}

Durch Einbringen von Distanzkörpern zwischen die Deckplatten der Sandwichstruktur können diese gegeneinander fest verspannt werden ohne das Kernmaterial zu belasten.

Es ist eine reine mechanische, formschlüssige Lösung, bei der der Kraftfluss genau definiert ist. Das Insert kann im Nachhinein in eine Sandwichplatte eingebaut werden. Es sind unterschiedliche Ausführungsvarianten für Schrauben, Bolzen, Lager und andere Maschinenelemente möglich.

Durch die feste Verschraubung von Grund- und Deckplatte der Sandwichstruktur können große Kräfte eingeleitet werden.

In Abbildung 3 wird eine Variante des Inserts gezeigt, die zum passgenauen Einbau eines Lagers in eine Sandwichplatte dient.

Bei der Herstellung von Sandwichplatten wird oft eine Wärmebehandlung benötigt, um das Kernmaterial mit den Deckplatten zu verbinden, bzw. die Deckplatten zu erhärten (CFK). Deshalb ist es nicht möglich Teile (Bolzen, Gewinde, Lager ...) passgenau schon bei der Herstellung der Sandwichplatten zu platzieren. Bei der Verwendung von Sandwichplatten als Halbzeug ist dies gar nicht möglich. 
Im Ausführungsbeispiel soll ein Kugellager betrachtet werden, welches passgenau in eine Sandwichplatte aus CFK eingefügt werden soll. Das Kugellager soll eine Zahnradwelle aufnehmen. Bei den Achsabständen sind Toleranzen von weniger $\frac{2}{100} \mathrm{~mm}$ notwendig.

Zuerst wird in das CFK-Sandwich eine passgenaue Bohrung eingebracht. Dies ist zum Beispiel mit CNC-Fräsen möglich. Die Bohrung und die Flanschteile 1 und 2 sind so toleriert, dass eine genaue Passung möglich ist. Weiterhin ist der Innenflansch 1 so toleriert, dass er den Außenring des Kugellagers genau führen kann.

Die Distanzkörper sind mit einer umlaufenden Feder verbunden. Diese werden in die Sandwichplatte eingebaut, in der vorher das Kernmaterial an der entsprechenden Stelle entfernt wurde. Die Flansche 1 und 2 werden gegeneinander fest verschraubt, sodass durch den Kraftschluss die beiden Deckplatten der Sandwichplatten und die Distanzkörper fest verschraubt sind. Der Innenflansch ist so gestaltet, dass das Kugellager 6 über Anschlag und Sicherungsring 8 eine klassische Festlagerung bildet.

a)

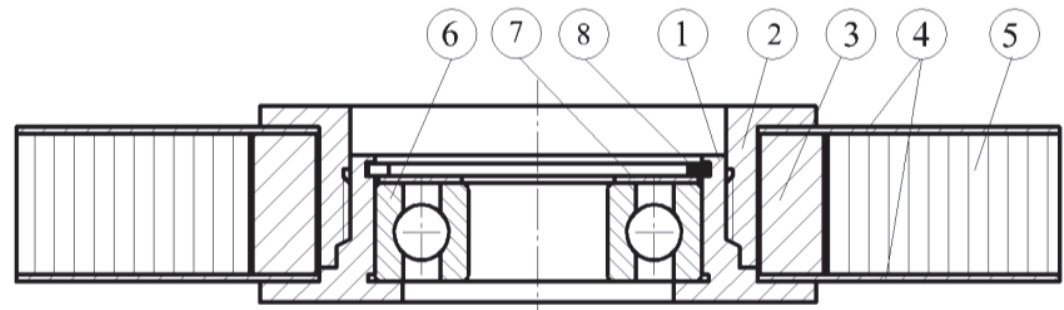

b)
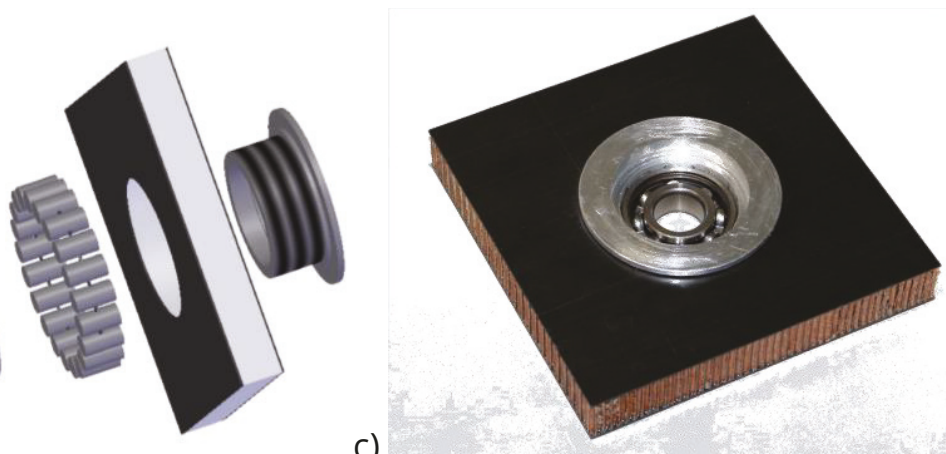

c)

Abbildung 3: Insertverbindung für Sandwichplatten nach Patent DE 102014014624 A1

a) Schnittdarstellung, b) Explosionsansicht, c) Prototyp 
Durch die patentierte Lösung wird es möglich große Kräfte in Sandwichplatten einzuleiten und diese Verbindungen im Nachhinein zu erstellen. Es können hohe Genauigkeiten erreicht werden, weil die Herstellung der Verbindung nach Abschluss aller Wärmebehandlung erfolgt und computergestützte Fertigungsverfahren zum Einsatz kommen.

Die Untersuchung des Prototyps der Insertverbindung, welcher gemäß der Beschreibung im Patent gefertigt wurde, ergab folgende Nachteile:

- Die Entfernung des Kernmaterials vor der Montage war schwierig und nur mit Spezialwerkzeug möglich,

- Die Montage der Distanzkörper ging ebenfalls schwierig,

- Die Insertverbindung wirkte im Vergleich zur Sandwichplatte nicht wirklich leicht.

An dieser Stelle wurde ein intensiver Ideenfindungsprozess gestartet, wobei das Brainstorming und das Analysieren die effektivsten Werkzeuge waren. Die Überprüfung der Machbarkeit der Ideen erfolgte mittels 3D gedruckter Modelle.

Die in Abbildung 4 dargestellten Modelle erweckten den Eindruck, dass alle Nachteile behoben werden konnten.
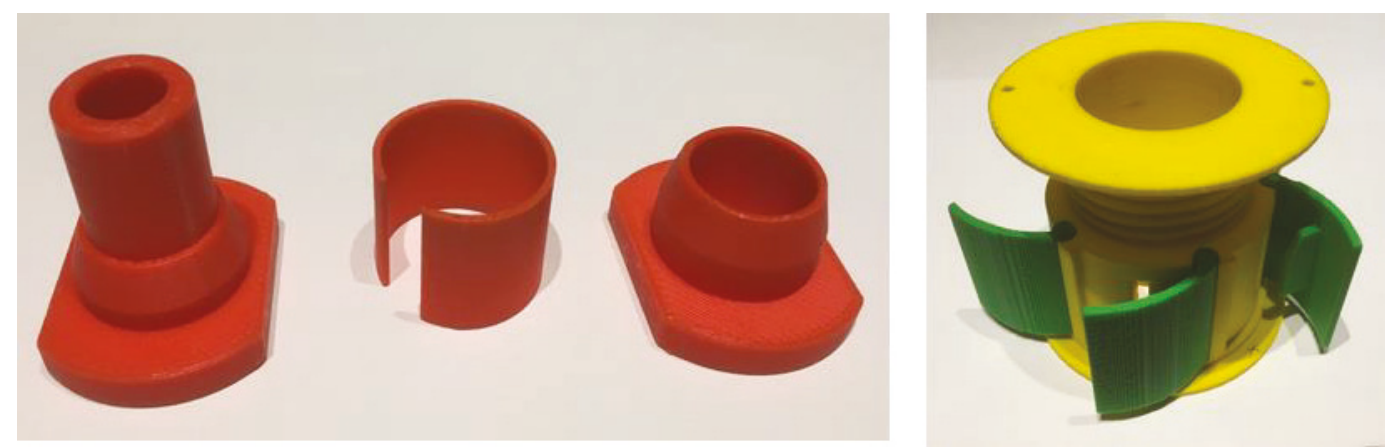

Abbildung 4: Insertverbindung 3D-Druckmodelle, links mit selbst aufspreizenden Distanzkörper, rechts mit durch gewindebetätigter Aufspreizung der Distanzkörper

So wurde Insertverbindung mit selbst aufspreizendem Distanzkörper gemäß dem Konstruktionsprinzip in Abbildung 4 links ein Prototyp hergestellt. Die einzelnen Bauteile sind in Abbildung 5 zu sehen. In Insertverbindung lässt sich gut montieren, ohne dass zuvor das Kernmaterial entfernt werden muss. 


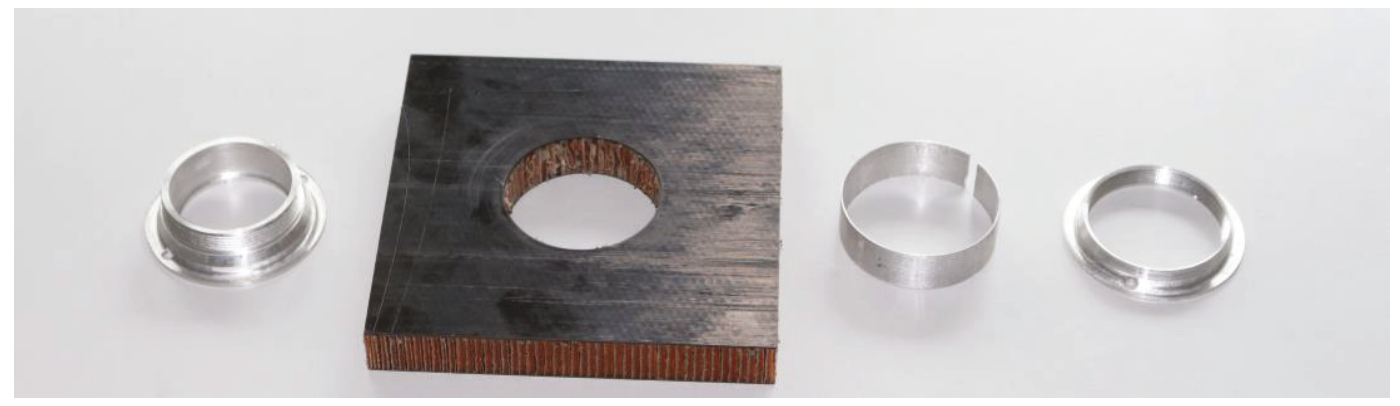

Abbildung 5: Insertverbindung für Sandwichplatten verbesserter Prototyp
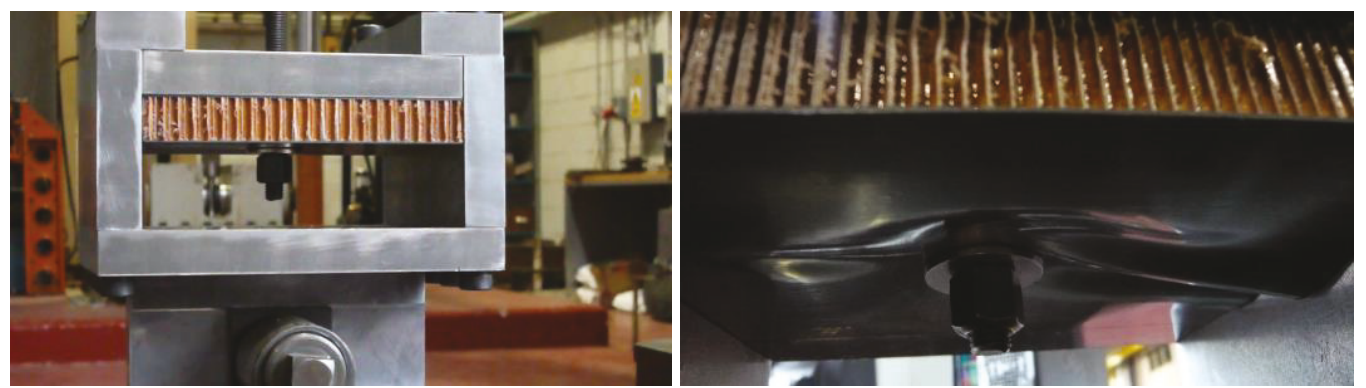

Abbildung 6: Insertverbindung für Sandwichplatten Ausreistest

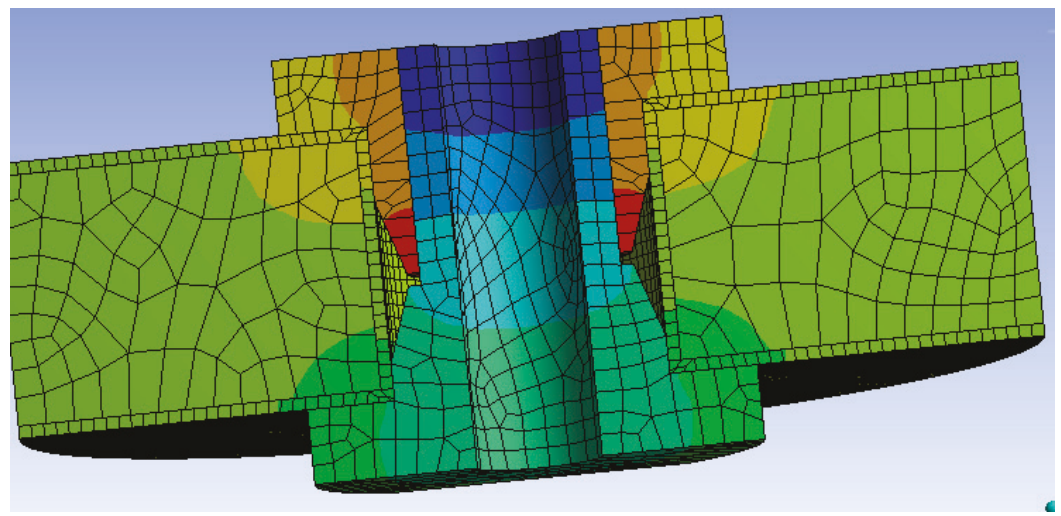

Abbildung 7: Insertverbindung für Sandwichplatten gemäß Abbildung 4 links FEM- Simulation der Verformungen 
Auf den ersten Blick wirkte die Verbindung auch stabil. Dies wurde genauer untersucht durch Ausreißversuche, siehe Abbildung 6 und Verifikation mit FEM- Berechnung, siehe Abbildung 7. Die Bauteilkomponenten wurden hinsichtlich ihrer Dimensionierung aufeinander abgestimmt. So passen die zulässige Lagerkraft, die durch die Insertverbindung aufnehmbare Kraft und die Stabilität der Sandwichplatte zusammen. So wurden, um mit dem Sprichwort zu sprechen, alle Glieder der Kette nahezu gleich stark gemacht. In der Praxis muss man dann gewisse Abstriche von diesem Prinzip machen um, die Teilevielfalt und die Größenabstufungen in praktikablen Grenzen zu halten.

Vergleichbare Insertverbindungen finden unter anderem Anwendung in dem Formula Student Fahrzeug der Hochschule Mittweida.

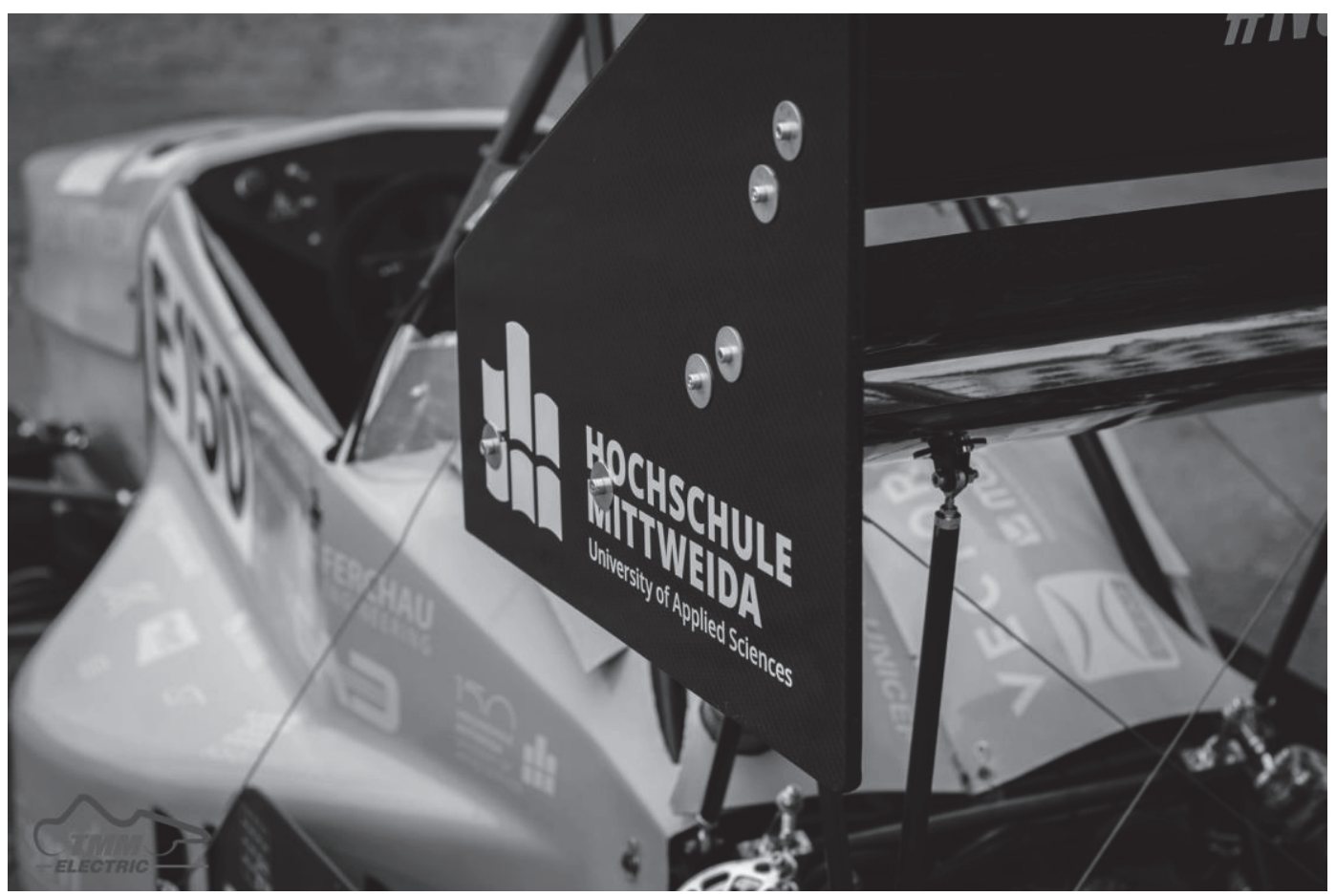

Abbildung 8: Formula-Student Fahrzeug 2019 der Hochschule Mittweida 


\section{Literaturverzeichnis}

Heimbs, S., \& Pein, M. (2009). Failure behaviour of honeycomb sandwich corner joints and inserts. Composite Structures, 89(4), 575-588.

VDI Richtlinie 2221,Methodik zum Entwickeln und Konstruieren technischer Systeme und Produkte. (1993), zurückgezogen (11.2019)

VDI Richtlinie 2221, Blatt 1: Entwicklung technischer Produkte und Systeme - Modell der Produktentwicklung (2019)

VDI Richtlinie 2221, Blatt 2:Entwicklung technischer Produkte und Systeme - Gestaltung individueller Produktentwicklungsprozesse (11.2019)

Frank Weidermann, Stefanie Zimmermann, Bob Bailey, David Smyth, Patentschrift Insert für eine Verbindung mit Sandwich Komponenten DE102014014624A1, veröffentlicht am 07.04. 2016

Zimmermann, S. (2019).A contribution to material specific design and analysis of carbon fibre reinforced plastic (CFRP). (PhD Thesis). University of the West of Scotland, Scotland.

\section{Kontakt}

Andrea Pino, M. Sc.

Dr. Stefanie Zimmermann

Prof. Dr. Ing. Frank Weidermann

Hochschule Mittweida

Fakultät Ingenieurwissenschaften

Technikumplatz 17

09648 Mittweida

www.hs.mittweida.de 